\title{
Relationship between circulating leptin and TNF $\alpha$ in obese and non-obese human subjects in the UK
}

\author{
M. Chaidas and L. S. Harbige \\ Centre for Biosciences Research, School of Science, University of Greenwich, Kent ME4 4TB, UK
}

\begin{abstract}
Obesity constitutes a worldwide health problem and is epidemic in many countries ${ }^{(1)}$ caused by accumulating fat to the point where health is likely to be affected. The objective of this study was to identify relationships between leptin, body fatness, BMI and the pro-inflammatory cytokine $\mathrm{TNF} \alpha$ in both obese and healthy control subjects. Ten healthy controls, mean age 23.3 years, mean BMI $<22.5 \mathrm{~kg} / \mathrm{m}^{2}$ and seven obese subjects, mean age 27.6 years, mean BMI $>34.5 \mathrm{~kg} / \mathrm{m}^{2}$ volunteered for the study. Body fat percentage was obtained with a 'Harpenden' skinfold calliper (sturdy metal construction with contoured handgrip) measurement in combination with a body composition programme (Jackson \& Pollock 4-Site Equation), the four sites measured were abdomen, suprailiac, triceps and thigh. Body fat percentage was measured using a futrex-5000 body composition analyser, inclusive of measuring ankle girth to measure frame. Plasma concentrations of leptin and the pro-inflammatory cytokine TNF $\alpha$ were measured in all subjects by ELISA (R\&D Systems, Ltd, Abingdon, UK). Both plasma TNF $\alpha$ and leptin concentrations were found to be significantly higher in the obese volunteers compared with healthy controls (Table)
\end{abstract}

\begin{tabular}{lcc}
\hline & Leptin $(\mathrm{pg} / \mathrm{ml})$ & TNF $\alpha(\mathrm{pg} / \mathrm{ml})$ \\
\hline Healthy controls $(n$ 10) & $379 \pm 274$ & $383 \pm 255$ \\
Obese subjects $(n$ 7) & $982 \pm 186^{*}$ & $756 \pm 200^{* *}$ \\
\hline$* P<0.001, * * P<0.01$. & &
\end{tabular}

Leptin correlated positively and significantly with BMI $(P<0.0001 ; r=0.72 ; n 17)$, and body fat $\%(P<0.0001 ; r=0.87 ; n 17)$. TNF $\alpha$ also correlated positively and significantly with BMI $(P<0.04 ; r=0.64)$ and positively with body fat $\%(P<0.06 ; r=0.60)$. There was an important trend indicating a positive relationship between plasma leptin concentration in both obese and control subjects and plasma $\mathrm{TNF} \alpha(P<0.58 ; r=0.59)$. This study indicates therefore that development of a pro-inflammatory state increases with body weight and percentage fat, as noted by others. There also appears to be a novel relationship between leptin and TNF $\alpha$ raising the question of whether leptin stimulates, e.g. monocytes to increase TNF $\alpha$ production, or TNF $\alpha$ induces adipocytes to produce leptin. The BMI independent relationship between leptin and TNF $\alpha$ is consistent with a regulatory role for leptin in the inflammatory state in obese subjects and would also be consistent with the notion that the TNF $\alpha$ cytokine network could be involved in the regulation of plasma leptin ${ }^{(2)}$. Future work needs to differentiate the biological effects of leptin and TNF $\alpha$ in obesity addressing the question of which is of primary importance, or if there is synergy between TNF $\alpha$ and leptin. The consequences of raised TNF $\alpha$ are not fully understood but the nutritional manipulation, e.g. $n-3$ fatty acids (20:5n-3 and 22:6n-3) or drug-based targeted therapy of TNF $\alpha$ could be potentially beneficial in obesity.

1. Dang M et al. (2010) Gasteroenterol Clin North Am 39, 1-7.

2. Chu N et al. (2000) Int J Obes Relat Metab Disord 24, 1085-1092. 